CSIRO group in Australia, which is led by Miss Turner and has included Dr Young, and many of their findings are reviewed in this book. Thus it follows to some extent the pattern of Lerner's Population Genetics and Animal Improvement which took examples from his work with poultry in California. The gap of 20 years between publication of the two books reflects the lag in application of the theoretical ideas to sheep.

The authors set out to describe the rationale of sheep improvement, and they conclude with specific recommendations for commercial breeding practice. The quantitative genetics is intended to fulfil a lesser role and is developed and applied only where necessary. The book could also be regarded as a text on quantitative genetics in which the examples are drawn predominantly from sheep. It does not fulfil this role adequately, however, for although most quantitative genetic and animal breeding principles are discussed, the depth of treatment depends on their importance in sheep production.

Much of the mathematical and statistical development is well presented, although the simplest proof is not always used. Several theoretical topics are analysed in unnecessary detail for such a text; for example, heritability formulae for ratio traits are derived in full. Similarly, lengthy formulae are given for selection indices on one trait using information from relatives, yet matrix algebra is introduced when discussing selection indices for several traits on one individual. The matrix methods should have been extended to include relatives' information so that more general results could have been obtained with less algebra. Some sections on applied topics also seem too detailed; for example, that on flock age structure, where the mathematics could have been collected and condensed. The discussion on mating systems including inbreeding is relatively short, and the authors clearly tread on unfamiliar ground. Here they make some errors of which the most misleading is an assertion that if in the cross between inbred lines "the $\mathbf{F}_{1}$ mean is outside the range of the parental means, overdominance and/or epistasis are important" (page 293).

The book is more limited in content than the title suggests, for the application is almost exclusively restricted to breeding merinos for wool production in Australia. While the geneties of meat production in sheep is less well understood it might have deserved more than passing mention, for even in the merino breed the authors suggest (page 1) that "meat production will become of greater importance".

The book is likely to be of greatest value as a review of the Australian research work. Clearly the publishers expected the market to be limited.

W. G. HrLI

\section{CHROMOSOMES AND LEUKAEMIA}

\section{Chromosome Studies in Acute Leukaemia}

By Mogens Krogh Jensen. Pp. 115. (Munksgaard: Copenhagen, July 1969.) $50 \mathrm{D}$ kr.

THIs monograph is a published version of the author's doctorate thesis, which probably accounts for the particular balance of its contents. The work begins with an elementary description of human chromosome nomenclature, the mechanisms of chromosoms aberration and the techniques for karyotypic examination of bone marrow and peripheral blood leucocytes. The literature on chromosome disorders in acute leukaemia is well reviewed. A wide variety of chromosome numbers and karyotypic patterns are encountered in this disease; about half the patients develop abnormal stem cell lines, but they are of little diagnostic or prognostic significance. The results of chromosome analyses of thirty acute leukaemic patients is the principal feature of this monograph. These and other analyses indicate that the myeloid and erythroid stem cells may have chromosome abnormalities similar to the leukaemic cells, a point of considerable importance when considering fundamental aspects of the disease. The value and pitfalls of interpretation in acute leukaemia of chromosome analyses of pəripheral blood leucocytes stimulated with phytohaemagglutinin, the effect of natural alterations of the relative numbers of leukaemic to non-leukaemic cells in the marrow and peripheral blood on the chromosome constitution of the population are discussed. The author rightly emphasizes some elementary biological factors that are easily forgotten when immersed in the technicality of karyotypic analysis. The remainder of the monograph is concerned with wider aspects of chromosomes and carcinogenesis, the incidence of leukaemia in patients with congenital karyotypic abnormalities and the influence of cytotoxic drugs.

This is a useful monograph, giving a fairly succinct, account of the present state of our knowledge of the disturbance of chromosome content and morphology in acute leukaemia. Unfortunately, the quality of the photomicrography is in places rather poor.

EDWARD H. COOPER

\section{APOPLEXY FOR EVERYMAN}

\section{Epidemiology of Cerebrovascular Disease}

By John F. Kurtzke. Pp. xv+197. (Springer-Verlag: Berlin, Heidelberg and New York, 1969.) DM58; \$14.50. STUDY of the patterns of ill health and death in populations which differ from each other in their geographical situations and ways of life has been most valuable in revealing genetic factors and constitutional and environmental hazards, which influence the appearance and development of disease in man. Taking this broad view of the purpose of epidemiology, Dr Kurtzke has used its methods to examine "strokes", one of the more fashionable human ailments.

In this short and very readable account, he first examines the bases of the current internationally accepted. method of classifying deaths caused by cerebrovascular disease, because the majority of epidemiological studies have relied on mortality statistics of uncertain validity. In his opinion, these types of data are adequate only for the whole group of strokes and, except in special circumstances, are too unreliable for the study of such specific causes as thrombosis of the cerebral arteries or cerebral haemorrhage. Data from several European countries, and the United States, have shown no significant local or regional differences in the incidence of cerebrovascular disease which cannot be attributed to the effects of diagnostic fashion and uneven distribution of medical care. He considers that the hypotheses that strokes are more common in Japan, and that they occur less often in Negroes, can be disproved by close analysis of the most reliable data. This chapter of the book illustrates the enormous and often unexpected difficulties which bedevil attempts to compare the incidence of disease in different countries. Intensive local surveys are often still the only way to obtain reliable information about morbidity and mortality, in spite of sophisticated analyses and attempted "correction" of national statistics.

The recent suggestion that there has been a significant change in the proportion of deaths caused by cerebral thrombosis and haemorrhage, which has led to much speculation about the possible pathogenetic roles of dietary and other exogenous factors, is also rejected because of what are considered to be flaws in the collection of the supporting evidence. The interpretation of Yates's findings, in particular, may appear far fetched, but the evidence available from other series is too vague to support this hypothesis.

Many of the factors involved in the causation of cerebral embolism and thrombosis, and of transient or minor strokes, are reviewed together with some aspects of the natural history of subarachnoid haemorrhage. 